\title{
Interkulturelle Kommunikation als wissenschaftliches Lehr- und Forschungsgebiet
}

\author{
Dr. phil. Olga Rösch
}

\section{Vortrags- und Diskussionsreihe „Inter- kulturelle Kommunikation" an der TFH Wildau}

Im Sommersemester 1997 startete das Sprachenzentrum der TFH Wildau eine Vortrags- und Diskussionsreihe unter dem Namen „Interkulturelle Kommunikation" (IK). Im Rahmen dieser Veranstaltungen soll unseren Studenten in Ergänzung zum Sprachunterricht die Möglichkeit geboten werden, Wissenswertes über andere Länder und ihre Kultur bereits während des Studiums an der Hochschule zu erfahren bzw. sich über ihre eigenen Erfahrungen im Ausland (z. B. im Praktikumssemester) auszutauschen. Es fanden schon Vorträge über China, Polen, Rußland, die USA, England, Irland, Korea, Japan und Kuba statt. Auf dem Programm des kommenden Semesters stehen weitere Beiträge über Frankreich, Spanien und andere Länder. Die Thematik der Vorträge reicht vom chinesischen Konfuzianismus über den politischen Konflikt in Irland, polnische Höflichkeitsetikette, deutsch-amerikanische Klischees auf beiden Seiten bis zur Problematik kultureller Mißverständnisse im allgemeinen. In den meisten Vorträgen wird auch dem fremdkulturellen Alltag ein gebührender Platz eingeräumt.

Im Wintersemester 1997/98 wird die Vortragsreihe im gleichen Veranstaltungsrahmen weitergeführt. Parallel dazu soll für Studenten des Studiengangs Betriebswirtschaft mit dem Schwerpunkt „Internationales Marketing" ein Seminar zum Thema IK angeboten werden. Im Seminar geht es um solche Fragen der IK, mit denen man bei einem intensiveren Kontakt mit Vertretern anderer Kulturen bzw. bei einem längeren (nicht touristischen) Aufenthalt in einer fremdkulturellen Umgebung unvermeidlich konfrontiert wird. Eine Auseinandersetzung mit diesen sog. „Begleiterscheinungen“ ist erforderlich, wenn die Kommunikation zu einem positiven Ergebnis (in welcher Form auch immer!) fuihren soll. Es werden also Themen behandelt wie Kulturschock und Kulturkonflikt, Stereotyp und Vorurteil, das Fremde und das Eigene, kulturelle Relativität der Wirklichkeit und Werte, Geschlechterrolle im Kulturvergleich sowie Begriffe wie Zeit, Raum, Symbol und Regel als soziale Kategorien werden erörtert. In Vorbereitung des Seminars ist im Sprachenzentrum bereits eine Bibliographie zur IK zusammengestellt worden, die Anfertigung einiger Reader für Studenten wird folgen. Während also den Schwerpunkt der Vortragsreihe die Vermittlung von Wissen und Anschauung über fremde Kulturen bildet, soll das Seminar einen Schritt weiter gehen und die Entwicklung der interkulturellen Handlungskompetenz fördern. Von
„Förderung interkultureller Handlungskompetenz“ wird deshalb gesprochen, weil davon ausgegangen wird, daß jeder Mensch grundsätzlich die Fähigkeit besitzt, fremde Kulturen verstehen zu lernen. Ziel des Seminars ist es, dieses Potential bewußt zu machen, zu verstärken und seinen Einsatz zu trainieren.

\section{Zum Kulturbegriff}

Es fällt auf, daß Begriffsbildungen wie „Interkulturelle Kommunikation“ und „Interkulturelle Kompetenz“ Anleihen am allgemeineren Begriff der "Kultur“ nehmen. Deshalb müssen wir uns zunächst fragen, welcher Kulturbegriff hier zugrunde zu legen ist.

In den Geistes,- Sozial- und Verhaltenswissenschaften gibt es kaum einen zweiten Begriff, der so oft und immer wieder aufs neue definiert wird wie der Kulturbegriff. Bereits vor vierundeinhalb Jahrzehnten haben Kroeber/Kluckhorn in ihrer vielzitierten Schrift „Culture“ rund 250 Definitionen des Begriffes ausgewertet und als Ergebnis eine Kulturdefinition präsentiert, die sie für erschöpfend hielten:

„Kultur besteht aus expliziten und impliziten Verhaltensmustern, die durch Symbole erworben und vermittelt werden, die spezifische Leistung einer menschlichen Gruppe begründen, einschließlich ihrer Verkörperung in Kulturprodukten. Der Wesensgehalt der Kultur besteht aus tradierten (historisch gewachsenen und selektierten) Ideen und damit verbundenen Wertvorstellungen. Kulturelle Systeme können einerseits als Ergebnisse von Handlungen und andrerseits als Bedingungselemente von Handlungen betrachtet werden." (Kroeber/ Kluckhorn, 1952:181)'

Die vorgeschlagene Definition hat die Kulturforscher jedoch nicht davon abgehalten, immer neue Definitionen des Kulturbegriffes auszuarbeiten. Der Psychologe A.Thomas (1993:379) geht davon aus, daß sich daran auch in der Zukunft nichts ändern wird. Er erklärt dies zu Recht damit, daß es keine einheitliche Vorstellung dessen, was unter Kultur zu verstehen ist, gibt und daß jeder darüber Schreibende „selbst seine Vorstellung von Kultur präzisiert, Kultur definiert und dadurch vermittelt, welche Aspekte dieses vielschichtigen Begriffes für ihn besonders bedeutsam sind." (ebd.).

Um nun zu verdeutlichen, was für uns „besonders bedeutsam" ist, wollen wir uns zunächst einen Definitionsversuch neueren Datums anschauen. Wie in jeder Abhandlung, die die Erscheinung Kultur - in welchem Kontext auch immer - zum Thema hat, finden wir somit 
auch erwartungsgemäß in „Kultur und Kulturwissenschaft" von K.Hansen (1995:9 f.) eine Beschreibung des Kulturbegriffs. Diese erachten wir in ihrem wissenschaftlich-methodischen Herangehen als ansprechend sowie auch als eine für einen Außenstehenden annehmbare Darstellung. Der Autor nähert sich dem komplexen Begriff uiber den Alltagsgebrauch des Wortes Kultur im Deutschen und nennt vier seiner Verwendungen:

1. Kultur als Bereich des gesellschaftlichen Lebens, vor allem das Umfeld von Literatur, Kunst, Musik u.ä., (,Kulturbetrieb“);

2. Kultur im Sinne einer bestimmten Lebensart: Manieren, Geschmack, Kunstsinn und Humanität, („Kultiviertheit");

3. Kultur als „way of life“; („das Brauchtum, die Sitten, die Religion").

4. Kultur als das Resultat einer Tätigkeit in der Landwirtschaft (z.B. „Monokultur“), in der Geographie („Kulturlandschaft"), aber auch in der Medizin („Bakterienkultur").

Der letzte, „äußerst prosaische Gebrauch“ (S.10) des Wortes soll uns hier nicht interessieren.

Die beiden ersten Verwendungen des Wortes sind nach Auffassung von Hansen beschreibend und wertend zugleich: beschreibend - weil sie jeweils ein Phänomen des Lebens bezeichnen; wertend - weil sie der ,praktischen Daseinsbewältigung... hochstehend übergeordnet" werden (vgl. S. 11). Dagegen sei die dritte Wortverwendung rein beschreibend, integriere aber die beiden ersten Bedeutungen des Wortes Kultur, meint der Autor. Sie erfasse (einem Fremdbetrachter auffallende) Eigenarten und Besonderheiten eines Volkes und sei wertneutral, „da jetzt die Gesamtheit der Gewohnheiten... im Vordergrund steht" (ebd.). Um diese integrierende Bedeutung des Kulturbegriffes geht es uns hier. Denn zum Wissen über eine fremde Kultur gehören nicht nur Informationen über Besonderheiten und Werte der Kulturträger, sondern auch ïber intrakulturelle Wertungen - betrifft vor allem die zweite Bedeutung von Kultur -, also über das kulturspezifische Orientierungssystem.

Zusammenfassend sei mir hier noch eine letzte Definition des Kulturbegriffes im Sinne von „way of life“ erlaubt, in der auch der fuir uns so wichtige kommunikative Aspekt angesprochen wird:

„Kultur ist die Gesamtheit der Gewohnheiten, Vorstellungen und Werte, die Menschen als Mitglieder einer Gesellschaft erworben haben und deshalb auch bei anderen Mitgliedern derselben erwarten (Hervorhebung von mir; O.R.), mit ihnen teilen“ (vgl. Weiße, 1994:4).

Man setzt also in der Kommunikation immer Sprachwissen und ein bestimmtes soziales Verhalten (bei den Vertretern des eigenen Kulturkreises) voraus, über das man sich nicht erst noch verständigen muß.

Ohne ein solches stillschweigend vorausgesetztes Wissen, d.h. ohne internalisierte Kultur, kann es keine Kommunikation geben. Wir machen uns kaum bewußt, wie sehr wir uns bei der Kommunikation darauf stützen, es sei denn, daß wir es ausdrüicklich trainiert haben, uns diese kulturelle Prägung bewußt zu machen. Wenn uns ein Mensch aus einer anderen Kultur gegenübertritt, werden wir zunächst dazu neigen, die von ihm nicht vorausgesetzten Vorstellungen und Werte unserer Kultur weiterhin unreflektiert zu gebrauchen. Durch ein interkulturelles Training sollen wir für die Wahrnehmung der in unserer Kommunikation enthaltenen kulturspezifischen Vorstellungen und Werte sensibilisiert werden und die Fähigkeit erlangen, die zur abweichenden Kultur unseres Partners gehörenden Vorstellungen und Werte zu entdecken und sie schrittweise mitverstehen zu lernen.

\section{Interkulturelle Kompetenz als Lernziel}

Die kulturellen Erwartungen beziehen sich auf sprachliche und nicht-sprachliche Handlungsmuster. Die Kommunikationspartner aus dem deutschsprachigen Raum erwarten z.B. in der Praxis der Geschäftsbeziehungen voneinander, daß jeder zu dem verabredeten Termin pünktlich erscheint, daß die gegenseitige Begrußung per Handschlag (unabhängig vom Geschlecht, Alter und Rang) erfolgt und daß jeder mit Herr bzw. Frau plus Familienname angesprochen wird usw.. Wenn dies nicht der Fall sein sollte, wird das abweichende Verhalten womöglich als „unpünktlich“, „unhöflich“, „familiär“, „komisch“ u.ä. (vorzugsweise negativ) bewertet. Treten wir in Kontakt mit Vertretern anderer Kulturen, z.B. aus dem arabischen Raum, so wird die Zahl dieser „Abweichungen“ rapide steigen. Auch wenn der gute Wille zur Verständigung auf beiden Seiten vorhanden ist, bedeutet eine Kooperation doch einen zuweilen nicht geringen Kräfteaufwand, denn man muß sich in einem fremdkulturellen Orientierungssystem zurechtfinden und sich dabei über sein eigenes bewußt werden, um für die andere Seite schließlich verständlich agieren zu können. Dies erfordert nämlich die spezifische Fähigkeit, , ïber eigene kulturelle Muster hinaus zu denken und zu handeln." (Kiel, 1996:84). Diese Fähigkeit wird mit dem Begriff der interkulturellen Kompetenz erfaßt und ist im geplanten Seminar als Lernziel anzustreben. Diese Fähigkeit der interkulturellen Kompetenz ist vor allem in einer fremdkulturellen Umgebung erforderlich, denn Verstöße gegen soziale Normen werden unvergleichbar härter bestraft als die Verletzung grammatischer Regeln in einer Fremdsprache.

Mit der Entwicklung von interkultureller Kompetenz im Landeskundeunterricht (vor allem aber mit der Vermittlung fremdkultureller Kompetenz für das Land der Zielsprache) beschäftigen sich Fremdsprachenphilologen seit längerem. So wurde u. a. für den Landeskundeunterricht ein aus mehreren Stufen zusammengesetztes Modell ausgearbeitet, dessen didaktisches Prinzip wie folgt beschrieben wird:

„Die Entwicklung interkultureller Kompetenz führt von der Sensibilisierung für die Problematik, über die kulturelle Sebstreflektion zur kulturellen Fremdreflektion und von dort zur Überprüfung dieser Reflektion in der Realität der Zielkultur." (Kiel, 1996:86) 
Das Beispiel oben mit dem Begrüßungsritual bei einem Geschäftstreffen ist gewiß ein relativ „oberflächliches“. Die Probleme der interkulturellen Kommunikation liegen zweifellos wesentlich tiefer und sind äußerst beziehungsreich. Deshalb ist es kein Zufall, daß verschiedene Wissenschaftsdisziplinen die Problematik der IK zu ihrem Forschungsgegenstand gemacht haben.

\section{Interkulturelle Kommunikation - ein interdisziplinäres Forschungsthema}

Wie bereits erwähnt, befassen sich also Fremdsprachenphilologen mit dem Thema IK im Rahmen der den Fremdsprachenunterricht begleitenden Landeskunde. Der Diskussions- und Forschungsstand zu Problemen der IK findet seit Jahrzehnten z. B. auch im Jahrbuch „Deutsch als Fremdsprache" seinen Ausdruck. Lehrveranstaltungen mit dieser Thematik sind inzwischen zum unverzichtbaren Bestandteil der Ausbildung von Fremdsprachenlehrern geworden. An einigen Hochschuleinrichtungen, vor allem deren Sprachenzentren ist die interkulturelle Forschung oft unter dem Namen „Fremdverstehen " institutionalisiert.

Wissenschaftler, die sich aus der Perspektive des Kulturtheoretikers mit der Problematik des Fremdverstehens befassen, sprechen von einer „Theorie der Interkulturalität" (vgl. Hansen, 1995:179 f.). Die rhetorische Frage „Wo aber findet man vorbildliche Forschungen für den Problemkreis Interkulturalität?“ (ebd. S. 188) läuft hier auf eine negative Bilanz hinaus: der Mangel an neuen „begriffsscharfen Konzepten“ wird allgemein beanstandet. So schreibt Hansen: „Weder verfügen wir über eine abgeschlossene und akzeptierte Vorstellung, wie Interkulturalität oder Fremdverstehen funktionieren könnte, noch besitzen wir genügend konkrete Forschungsergebnisse über erfolgreiche oder erfolglose Verständigung dieser Art.“ (S. 180). Der Begriff „Interkulturalität" selbst scheint mir überdies auch nicht besonders produktiv und hilfreich zu sein, denn er suggeriert eine Vorstellung, als gäbe es sozusagen einen Raum über den Kulturen.

Die Fragen der IK beschäftigen auch Sozial- und Erziehungswissenschaftler. Anfang der 80er Jahre wurde in Deutschland unter dem Einfluß der anglo-amerikanischen Migrationsforschung und durch Übernahme einiger Erziehungskonzepte der Begriff „Interkulturelle Erziehung" geprägt. Seitdem reißt die Flut von Arbeiten nicht ab, die diesen Begriff in ihrem Titel führen (vgl. z.B. Hohmann/Reich, 1989; Jahrbiicher für interkulturelles Lernen). In einem großen Teil der Veröffentlichungen bekommt die Vokabel interkulturell einen „politisch-programmatischen Bedeutungsgehalt“ (Schweizer, 1994:12). Somit erfüllen die Schriften eher eine ideologische Funktion. Die Publikationen zur interkulturellen Erziehung geraten aber allzuoft zu „Klageschriften“. Auch der sonst im Grunde positiv besetzte Begriff „Interkulturelle Kommunikation“, den wir gern mit Bemühungen um die Völkerverständigung assoziieren, verliert in der Studie von H.Schweizer (1994) zu sozialwissenschaftlichen Grundlagen interkultureller
Erziehung an Aura, wenn der Autor die IK als „Machtstrategie in der Geschichte“ (ebd. S. 17 f.) betrachtet. Noch nüchterner klingt er im Zusammenhang mit der Kolonialschuld (vgl. Mühlhäusler, 1990).

Auch Psychologen widmen sich der Thematik der IK im Rahmen der sog. „Kulturvergleichenden Psychologie“ (vgl. Thomas (Hrg.), 1993) und forschen nach den Ursachen konflikthafter Interaktionen. Die Psychologen leisten damit einen wesentlichen Beitrag zur Entwicklung von interkulturellen Trainingsprogrammen zur Förderung interkultureller Managementkompetenzen für Fach- und Führungskräfte aus der Wirtschaft (vgl. Thomas/Hagemann, 1992).

Wenn in diesem - keineswegs erschöpfenden - Überblick über die Behandlung von IK in unterschiedlichen Disziplinen die Ethnologie/empirische Kulturanthropologie an letzter Stelle aufgefuihrt wird, so driickt dies leider ein Dilemma aus. Als einzige Disziplin hat sie eine Methodik der umfassenden Beschreibung von Kultur, nämlich die Ethnographie, entwickelt. Von Vertretern dieser Disziplin wurde eine Fülle an empirischem Material zusammengetragen. Leider sind aber Beiträge von Ethnologen/ Kulturanthropologen zu IK und zu interkulturellem Training bisher eher die Ausnahme als die Regel.

Der Forderung der Wirtschaft nach angemessenen Qualifizierungsangeboten für ihre Fach- und Führungskräfte stellten sich viele Fachleute aus verschiedenen Wissensgebieten. Im Unterschied zu (Geistes)Wissenschaften hat die Wirtschaft in dieser Frage äußerst praktisch orientierte Ansätze und Ziele:

„Stets geht es um eine Optimierung der Zusammenarbeit zwischen Mitgliedern verschiedener Nationalkulturen, von der Kostensenkung und Umsatzsteigerung erwartet wird." (Hansen, 1995:188).

Diesem Ziel folgend entwickelte sich inzwischen als Forschungsfeld die „Interkulturelle Wirtschaftskommunikation". Hier fließen Erkenntnisse, Konzepte und Methoden aus vielen wissenschaftlichen Disziplinen, vor allem aber auch aus der Praxis zusammen (vgl. Interkulturelle, 1994). Im Rahmen dieser Forschungen befaßt man sich mit sprachlich und kulturell bedingten Störungen in der Kommunikation bei internationalen Wirtschaftsbeziehungen. Die Aufgabe der neuen Disziplin besteht darin, „systematisch und interdisziplinär zu analysieren, welche Faktoren die grenzüberschreitende Kommunikation in Handlungsfeldern der Wirtschaft negativ oder positiv beeinflussen." (vgl. Müller, 1991). Die Arbeit auf diesem Gebiet wird u.a. vom Institut für Auslandsbeziehungen unterstützt und in Veröffentlichungen dokumentiert (vgl. Interkulturelle, 1993).

Wir erleben heute an verschiedenen Hoch- und Fachhochschulen erste Ansätze für eine ergänzende Ausbildung in einem Fach wie „Interkulturelle Kommunikation“ u.ä.. Bezieht man die sich rasch vollziehenden Veränderungen in Europa und in der gesamten Welt mit in die Betrachtung ein, so ist anzunehmen, daß diese Fächer schnell an Bedeutung zunehmen werden. Vermutlich wird es nicht mehr lange dauern, bis eine ergänzende Ausbildung in 
IK als obligatorische Schliisselqualifikation angesehen werden wird. Um den Studierenden unserer Fachhochschule hierzu den Zugang zu eröffnen, wurden die zuvor beschriebenen Lehrveranstaltungen in das Lehrprogramm aufgenommen. Von dieser sinnvollen Zusatzqualifikation können unsere Studenten und Absolventen sowie Teilnehmer an Weiterbildungskursen in ihrem späteren Berufsleben nur profitieren.

\section{Literatur}

Hansen, Klaus P. (1995):

Kultur und Kulturwissenschaft. Eine Einführung. Tübingen und Basel.

Hohmann, Manfred/Reich, Hans H. (Hrg) (1989):

Ein Europa für Mehrheiten und Minderheiten. Diskussionen um interkulturelle Erziehung. Münster/New York.

Interkulturelle Kommunikation und der Auslandseinsatz von Fach- und Führungskräften (1993). Annotierte Literaturauswahl. Schriftenreihe des Instituts für Auslandsbeziehungen, Reihe Dokumentation, Bd. 20, Stuttgart.

Interkulturelle Kommunikation und interkulturelles Training. Problemanalysen und Problemlösungen (1994). Materialien zum Internationalen Kulturaustausch. Hrg.: Institut für Auslandsbeziehungen, Bd. 33, Stuttgart.

Kiel, Edward (1996):

Kulturanalyse im Landeskundeunterricht als Mittel der Entwicklung interkulturellen Kompetenz. In: Fremdsprachen und Hochschule, 46, S.82-101.

Mühlhäusler, Peter (1990):

Interkulturelle Kommunikation - cui bono? In: Spillner, B. (Hrg.): Interkulturelle Kommunikation. Kongreßbeiträge zur 20. Jahrestagung der Gesellschaft für Angewandte Linguistik, S.19-29, Frankfurt am Main/u.a.

Müller, Bernd-Dietrich (Hrg.) (1991):

Interkulturelle Wirtschaftskommunikation. München.

Schweizer, Helmuth (1994):

Der Mythos vom interkulturellen Lernen. Reihe Studien zu Migration und Minderheiten, Hrg.: D. Thränhardt, Bd.2, Münster/Hamburg.

Thomas, Alexander (1993):

Psychologie interkulturellen Lernen und Handels. In: Ders. (Hrg.): Kulturvergleichende Psychologie. Eine Einfuihrung. S. 377-424, Göttingen.

Thomas, Alexander/Hagemann, Katja (1992):

Training interkultureller Kompetenz. In: Bergemann, Niels und Andreas L.J. Sourrisseaux (Hrg.): Interkulturelles Management. S. 173-199, Heidelberg.

Weiße, Frieder (1994):

Kontinuität und Wandel. Aspekte türkischer Kultur. Berlin.

\section{Verfasserin}

Dr. phil. Olga Rösch

Leiterin des Sprachenzentrums an der TFH Wildau

Tel.: 03375/508 367 oder.../508348.

\section{Fußnote}

1 Der deutsche Text wird nach A. Thomas (1993:379) zitiert. Original: Kroeber, A.A/Kluckhorn,C.: Culture: A critical review of concepts and definitions. Cambridge, MA: Peabody Museum, Vol. 47, No. 1. 